\section{Obesidad y cáncer - Los dos escenarios que Chile liderará}

\section{Obesity and Cancer - The two scenarios that Chile will lead}

\section{Sr. Editor:}

Actualmente, 7 de cada 10 adultos chilenos presenta malnutrición por exceso. De ellos, más del $30 \%$ posee obesidad según datos de la última Encuesta Nacional de Salud 2016-2017ㄹ. Lamentablemente, esta realidad no está ajena a la población infantil; de acuerdo al último mapa nutricional 2018, uno de cada cuatro niños en edad preescolar ha sido diagnosticado con obesidad ${ }^{2}$. Sin embargo, el problema no termina ahí, ya que la obesidad no sólo es un importante factor de riesgo para el desarrollo de enfermedades cardiovasculares, sino también posee una alta relación con el desarrollo de cáncer. Así fue reafirmado por el último reporte de "World Cancer Research Found (WCRF)", , donde la obesidad y el sobrepeso fueron asociados con un mayor riesgo de desarrollar 12 tipos diferentes de cánceres, entre ellos cáncer de mama, próstata, colorrectal y vesícula biliar (Figura 1b). Estos cuatro cánceres mencionados son los que presentan actualmente la mayor prevalencia en Chile (Figura 1a). Se estima que actualmente en Chile el número de muertes por cánceres es el doble que las muertes atribuidas a enfermedades cardiovasculares
(ECVs) (razón entre muertes por ECVs/Cáncer = 0,41), escenario que se asimila la realidad de países de alto ingreso económicos como Canadá y Suecia, donde 23\% de todas las muertes son debido a ECVs, mientras que un $55 \%$ son debido a cáncer ${ }^{4}$. En este contexto y considerando las alarmantes tasas de obesidad en población chilena -un factor de riesgo clave en el desarrollo de cáncer- es relevante conocer el escenario epidemiológico al que nos enfrentamos como país ${ }^{5}$.

El último reporte del WCRF señala que la prevención es fundamental para reducir el riesgo de desarrollar cáncer, siendo el estilo de vida el principal factor protector o de riesgo. Entre las principales recomendaciones del WCRF relacionadas a un estilo de vida saludable están: mantener un peso corporal saludable; ser físicamente activo; adoptar una dieta rica en granos enteros, vegetales, frutas y legumbres; limitar el consumo de comida rápida y carnes, especialmente carnes rojas y procesadas; limitar el consumo de bebidas azucaradas y el alcohol. Sin embargo, la proporción de la población chilena que cumple con estas recomendaciones es baja, por lo cual el escenario para el riesgo de cáncer en Chile no es alentador. Según datos de la Encuesta Nacional de Consumo Alimentario (ENCA), solo 52\% de los chilenos cumple con las recomendaciones de consumo de frutas y verduras, $25 \%$ con la recomendación de consumo de legumbres; no obstante, $44 \%$ de los individuos consume cereales procesados, $95 \%$ de la población consume carnes rojas y procesadas y más del $90 \%$ reporta consumir azúcar y bebidas azucaradas, lle-

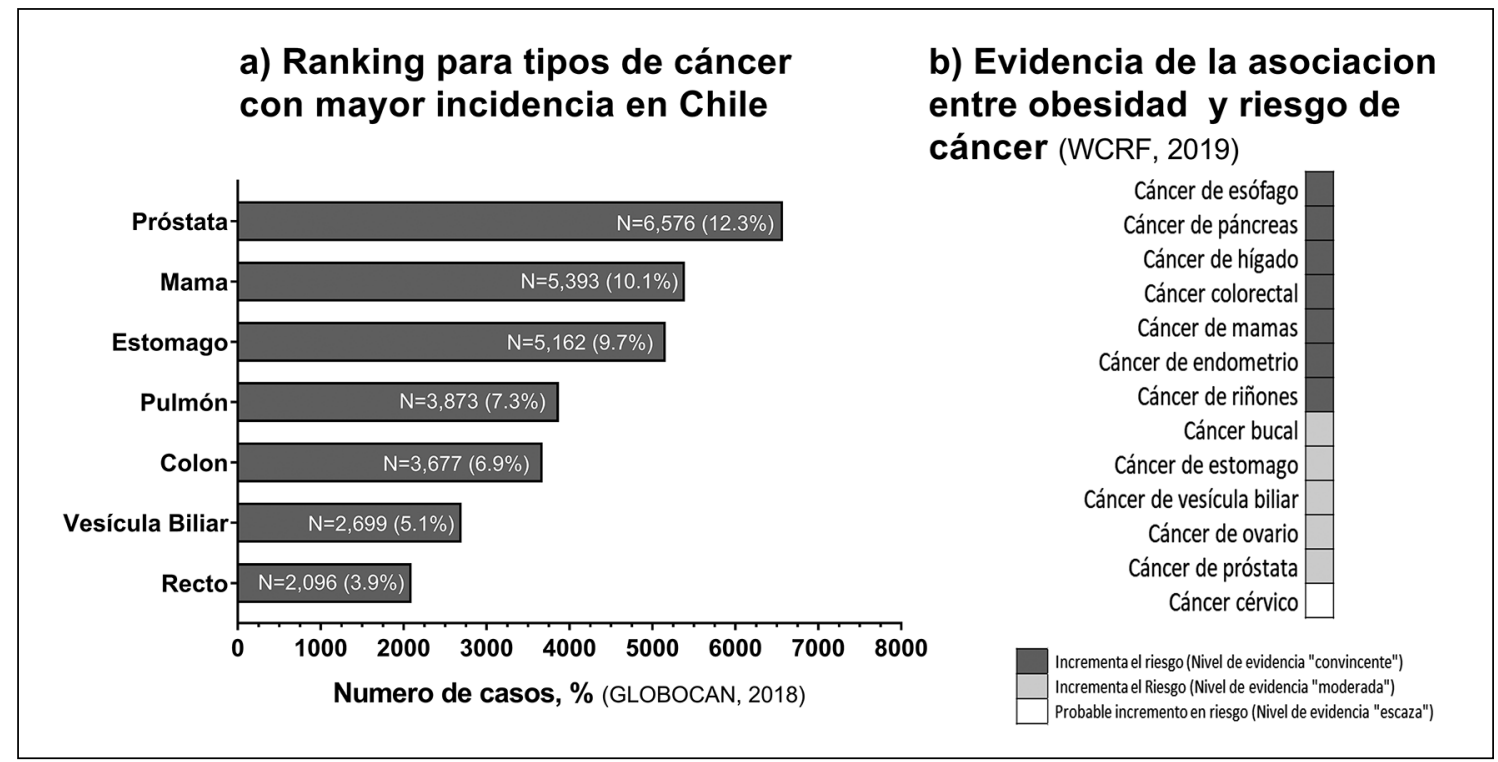

Figura 1. Ranking de los cánceres más prevalentes en Chile (a) y lista de canceres según su grado de asociación con obesidad (b). Los datos de la figura (a) fueron extraídos del reporte de GLOBACAN-2018 y son presentados como número de casos y su respectiva prevalencia según tipo de cáncer. Los datos de la Figura (b) fueron extraídos del último reporte de la WCRF-2019. 
gando a un promedio de $20 \mathrm{~g} /$ día de azúcares ${ }^{6}$. Además, a este escenario se suma que más de $89 \%$ de los chilenos reportan ser físicamente inactivo en su tiempo libre ${ }^{1}$.

Para cumplir con las recomendaciones sugeridas por el WCRF en la prevención del riesgo de desarrollar cáncer, se requieren políticas públicas orientadas a promover los estilos de vida saludable y modificar aquellos factores de riesgo que se encuentran presentes en la población chilena. Seguir una alimentación saludable y realizar actividad física va más allá de decisiones individuales; es también un compromiso por parte del Estado en la inversión de infraestructura que modifique el entorno en el que vive actualmente nuestra población. Algunas de estas medidas incluyen la restricción de alimentos no saludables como productos procesados y altos en grasas saturadas o azúcar, incorporación de programas de incentivo como la reducción de impuestos para acceso a alimentos saludable como pescados grasos, frutos secos, frutas y verduras, como también así una mayor inversión en la construcción de ciclovías, parques y áreas verdes que contribuyan a aumentar los niveles de actividad física de la población. Estas medidas podrían ayudar a reducir la alta prevalencia de estos factores de riesgo asociados a cáncer, especialmente a través de la disminución de los niveles de obesidad en nuestra población. Si las tendencias continúan y no existen políticas públicas que apoyen el cambio de conductas y estilos de vida, sin duda el cáncer se posicionará dentro de la próxima década como la enfermedad asociada a mayor mortalidad en el país.

Solange Parra-Soto ${ }^{1}$, María Adela Martínez-Sanguinetti ${ }^{2}$, Fanny Petermann-Rocha ${ }^{1}$, Carlos Celis-Morales ${ }^{3}$. ${ }^{1}$ Institute of Health and Wellbeing, University of Glasgow, Glasgow, United Kingdom. ${ }^{2}$ Instituto de Farmacia, Facultad de Ciencias, Universidad Austral de Chile. Valdivia, Chile. ${ }^{3}$ BHF Glasgow Cardiovascular Research Centre Institute of Cardiovascular and Medical Science,
University of Glasgow, Glasgow, United Kingdom.

\section{Referencias}

1. Ministerio de Salud de Chile. Encuesta Nacional de Salud 2016-2017. Web: https:/www.minsal.cl/wp-content/ uploads/2017/11/ENS-2016-17_PRIMEROS-RESULTADOS.pdf (Accedido el 12 de septiembre de 2019).

2. JUNAEB. Mapa Nutricional. Web : https://www.junaeb. $\mathrm{cl} /$ mapa-nutricional. (Accedido el 8 de septiembre de 2019).

3. World Cancer Research Found/ American Institute for Cancer Research. Diet, Nutrition, Physical Activity and Cancer: A Global Perspective. Continuous Update Project Expert. (2018). Web: https://www.wcrf.org/dietandcancer (Accedido el 12 de septiembre de 2019).

4. Dagenais GR, Leong DP, Rangarajan S, Lanas F, López-Jaramillo P, Gupta R, et al. Variations in common diseases, hospital admissions, and deaths in middle-aged adults in 21 countries from five continents (PURE): a prospective cohort study. The Lancet 2019, pages 1-10. https:/doi. org/10.1016/S0140-6736(19)32007-0.

5. Ministerio de Salud. Plan Nacional de Cáncer 2018-2028. Web: https://www.gob.cl/plannacionaldecancer/ (Accedido el 12 de septiembre de 2019).

6. Ministerio de Salud de Chile. Encuesta Nacional de Consumo Alimentario. Web: https://www.minsal.cl/enca/ (Accedido el 12 de septiembre de 2019).

Correspondencia a:

Carlos Celis-Morales

Institute of Cardiovascular and Medical Sciences, University of Glasgow, Glasgow, United Kingdom.

Carlos.Celis@glasgow.ac.uk 